\title{
Expression of the Histidine Kinase Gene Sshk Correlates with Dimethachlone Resistance in Sclerotinia sclerotiorum
}

\author{
Jinli Li, Fuxing Zhu, ${ }^{\dagger}$ and Jianhong $\mathrm{Li}^{\dagger}$
}

College of Plant Science and Technology, Huazhong Agricultural University, Wuhan, 430070, China. Accepted for publication 27 July 2018.

\begin{abstract}
Histidine kinases (HK) are implicated in virulence, vegetative mycelial growth, and osmotic and oxidative responses in pathogenic fungi. Our previous work showed that transcriptional levels of the group III HK gene Sshk are higher in field dimethachlone-resistant isolates of Sclerotinia sclerotiorum compared with sensitive isolates. However, it is not clear whether the overexpression of $S s h k$ is the major mechanism for resistance to dimethachlone. In this study, we constructed $S s h k$ silencing and overexpression vectors and assessed dimethachlone resistance levels, virulence, mycelial growth, and sensitivity to osmotic stress for the $S s h k$-silenced and -overexpression transformants. Overexpression of Sshk resulted in resistance to dimethachlone and increased sensitivity to various stresses and to the cell-wall-perturbing agents sodium dodecyl sulfate (SDS) and Congo red (CR). Compared with the parent isolate,

Ssh $k$-silenced transformants had reduced resistance to dimethachlone, significantly higher $(P<0.05)$ mycelial growth and virulence, and lower sclerotium production, and were less sensitive to various exogenous stresses such as sodium chloride. Compared with the parent sensitive isolate HLJMG1, dimethachlone resistance ratios of the three overexpression transformants $\Delta \mathrm{C} 101, \Delta \mathrm{C} 21$, and $\Delta \mathrm{C} 10$ increased 168.1-, 189.5-, and 221.2-fold, respectively. The three overexpression transformants were more sensitive to CR and SDS than their parent isolate. These findings suggest that overexpression of $S s h k$ is a major mechanism for dimethachlone resistance in some isolates of S. sclerotiorum, and that Sshk plays an important role in maintaining the integrity of the cell wall. Our findings reveal a novel molecular mechanism for dimethachlone resistance in plant-pathogenic fungi.
\end{abstract}

Sclerotinia sclerotiorum (Lib.) de Bary is a necrotrophic fungal plant pathogen with a worldwide distribution capable of infecting over 400 species of plants, leading to severe economic losses in the production of oil crops and vegetables (Bardin and Huang 2001; Bolton et al. 2006; Letham et al. 1976). Due to the lack of genetic resources that could be utilized in crop breeding programs, the control of $S$. sclerotiorum has been relying heavily on the application of fungicides (Bolton et al. 2006). The benzimidazole fungicide carbendazim had been widely used to control S. sclerotiorum in China prior to 2000, and high levels of carbendazim resistance were reported in the late 1990s in East China (Pan 1998). Since the early 2000s, the dicarboximide fungicide (DCF) dimethachlone has been widely used to control S. sclerotiorum in China (Kuang et al. 2011). Due to repeated applications of dimethachlone, medium to high levels of dimethachlone resistance have been reported subsequently in China (Ma et al. 2009; Zhou et al. 2014a,b). Previous studies indicate that the group III histidine kinase (HK) genes that function within a twocomponent signal transduction pathway are involved in DCF resistance in several filamentous fungi (Alex et al. 1996; Cui et al. 2002; Duan et al. 2013; Luo et al. 2012; Motoyama et al. 2005).

The two-component signal transduction system consists of an $\mathrm{HK}$ domain and a response regulator (RR) domain. Phosphorylation of histidine of $\mathrm{HK}$ and aspartic residues of RR leads to the activation of a signal transduction pathway, resulting in the recruitment of downstream mitogen-activated protein kinase cascades (West and Stock 2001). In pathogenic yeast and fungi, the two-component pathway mediates osmoregulation, mycelial development, and cell

†Corresponding authors: Jianhong Li; E-mail: jianhl@mail.hzau.edu.cn; and Fuxing Zhu; E-mail: zhufuxing@mail.hzau.edu.cn

Funding: This study was supported by the National Natural Science Foundation of China (31371964).

(c) 2019 The American Phytopathological Society growth (Calera et al. 2000; Li et al. 1998; Srikantha et al. 1998; Thomason et al. 1999; Wurgler-Murphy and Saito 1997). The twocomponent signal pathway in filamentous fungi is similar to but more complex than that of budding yeast because the latter has a sole HK gene, SLN1, and the deletion of this gene is lethal (Posas et al. 1996). Compared with yeast, filamentous fungi have a diverse number of HK genes (Ochiai et al. 2002; Tanaka and Izumitsu 2010). The putative HK genes are categorized into 11 groups (Catlett et al. 2003), of which group III HK lacks a transmembrane structure and is primarily involved in the response to high osmolarity (Duan et al. 2013; Izumitsu et al. 2010; Luo et al. 2012; Tanaka and Izumitsu 2010; Yoshimi et al. 2004) and in virulence (Cho et al. 2009; Liu et al. 2008; Luo et al. 2012; Rispail and Pietro 2010; Viaud et al. 2006), conidial morphology (Viaud et al. 2006), and cell wall integrity (Zhang et al. 2010) of phytopathogenic fungi. Numerous studies have demonstrated that mutations of group III HK genes are responsible for DCF resistance in filamentous fungi, and these $\mathrm{HK}$ genes include $\mathrm{BcOS} 1$ in Botrytis cinerea, os-1 in Neurospora crassa, and Dicl in Bipolaris maydis (Cui et al. 2002; Ochiai et al. 2001; Oshima et al. 2002; Yoshimi et al. 2004). In Pyricularia grisea, S. sclerotiorum, and Alternaria longipes, deletion of the group III HK genes HIK1, Shkl, and AlHK11, respectively, leads to resistance to DCF and phenylpyrrole fungicides (Duan et al. 2013; Luo et al. 2012; Motoyama et al. 2005). In addition, lost expression of HK gene $\mathrm{SsOs} 1$ may be involved in dimethachlone resistance in S. sclerotiorum (Firoz et al. 2016). These studies indicate that the group III HK genes play an important role in DCF resistance in various filamentous fungi.

Our previous studies showed that the expression levels of the group III HK gene $S s h k$ are higher in field dimethachlone-resistant isolates than in sensitive ones of S. sclerotiorum (Li et al. 2017) but there is no direct evidence to show that increased transcription of the gene $S s h k$ is the major mechanism for resistance to DCF. In the current research, therefore, we constructed $S s h k$-silencing and $S s h k$ overexpression vectors to determine the correlation between $S s h k$ expression and dimethachlone resistance in S. sclerotiorum. 


\section{MATERIALS AND METHODS}

Isolates and culture conditions. The dimethachlone-resistant S. sclerotiorum isolate HLJ4 and the sensitive isolate HLJMG1 were used in the present study, and their effective concentrations for $50 \%$ inhibition of mycelial growth $\left(\mathrm{EC}_{50}\right.$ values) were 38.45 and $0.18 \mu \mathrm{g} / \mathrm{ml}$, respectively (Li et al. 2017). Surface-sterilized sclerotia were placed on potato dextrose agar (PDA) dishes and incubated at $23^{\circ} \mathrm{C}$ in the dark for 2 days, mycelial plugs $(5 \mathrm{~mm}$ in diameter) were cut from the fresh edge of 2-day-old colonies and transferred to fresh PDA dishes, and the resulting colonies were used for subsequent experiments. Escherichia coli strain TOP10 (Weidi Biotechnology, Shanghai, China) was used to propagate all plasmids, and Agrobacterium tumefaciens strain EHA105 (Weidi Biotechnology) was used for transformation of S. sclerotiorum. The $S s h k$-silenced transformants were cultured on PDA media amended with hygromycin B at $30 \mu \mathrm{g} / \mathrm{ml}$ (Aladdin Biotech, Shanghai, China), and the $S$ shk-overexpression transformants were cultured on PDA media amended with geneticin G418 at $40 \mu \mathrm{g} / \mathrm{ml}$ (Aladdin Biotech).

Construction of $\boldsymbol{S s h} \boldsymbol{k}$-silencing and -overexpression vectors. Sshk-silencing and -overexpression vectors were constructed according to published protocols (Yu et al. 2012), with minor modifications. For the construction of the $S s h k$-silencing vector, a 306-bp fragment from $S s h k$ was amplified with primers SSHKF and SSHKR (Table 1) from $S$. sclerotiorum cDNA and ligated to the TEasy plasmid (Promega Biotech, Beijing, China) by T4 DNA ligase to produce the Sshk-4 plasmid. The plasmid Sshk-4 was digested with restriction endonucleases PstI and BamHI (Takara Biotech, Beijing, China) and the target fragment was subsequently ligated into the digested pCIT vector (Yu et al. 2012) to produce a pCIT1 vector. Subsequently, the plasmid Sshk-4 was digested with HindIII and ClaI (Takara Biotech) and the target fragment was ligated into the digested pCIT1 to produce a pCIT2 vector. The vector pCIT2 containing the two $S s h k$ gene fragments in the opposite orientation was digested with $\mathrm{XhoI}$ and SacI (Takara Biotech) and ligated into the $\mathrm{pCH}$ plasmid to generate an Sshk-SM silencing vector.

For the construction of the $S s h k$-overexpression vector, a $3.9-\mathrm{Kb}$ full-length $S s h k$ gene was amplified with primers OEHKF and OEHKR (Table 1) from $S$. sclerotiorum cDNA and then ligated into the digested pCETNS-4 vector (Yang et al. 2018) at the SacI and SmaI (Takara Biotech) sites to generate the overexpression vector Sshk-OE. All constructs were assessed by sequencing analysis and introduced into A. tumefaciens EHA105 (Weidi Biotechnology) by the electroporation-mediated method (Yu et al. 2012).

Transcription levels of the $S \boldsymbol{s h} \boldsymbol{k}$-silenced and -overexpression transformants. Mycelial plugs (5 $\mathrm{mm}$ in diameter) cut from the edge of 2-day-old colonies were transferred to PDA dishes overlaid with cellophane (Bo'ao Biotechnology), and mycelia of each isolate were harvested after $48 \mathrm{~h}$ of incubation at $23^{\circ} \mathrm{C}$. Genomic DNA was extracted according to the manufacturer's instructions
(HP Fungal DNA kit; OMEGA Biotek, Shanghai, China), and TDNA insertions in different transformants were examined by polymerase chain reaction (PCR) using the primers Hyg-F: CAGCGTCTCCGACCTGAT and Hyg-R: TCTGCGGGCGATT TGT (Jiang 2007). To evaluate transcriptional levels of $S s h k$ in different transformants, total RNA from each isolate was extracted using TRIZOL Reagent (Invitrogen, Carlsbad, CA). First-strand cDNA was synthesized and quantitative reverse-transcription (qRT)-PCR was conducted as previously described (Li et al. 2017). Transcriptional levels of $S s h k$ were evaluated by qRT-PCR using the primers RT-F/R (Table 1) and normalized to the $\beta$-tubulin gene using the primers $\beta$ tub-F/R (Table 1), and the relative levels of gene transcription were calculated with the $2^{-\Delta \Delta \mathrm{Ct}}$ method (Livak and Schmittgen 2001). Three replicates for each isolate were analyzed and the experiment was independently repeated three times.

Determination of dimethachlone sensitivity. Dimethachlone sensitivity of the $S s h k$-silenced transformants and the parent isolate HLJ4 was measured on PDA amended with dimethachlone at $0,10,20,30,40,50$, and $60 \mu \mathrm{g} / \mathrm{ml}$, whereas dimethachlone at 0,5 , $10,20,40,60$, and $80 \mu \mathrm{g} / \mathrm{ml}$ was used for the $S s h k$-overexpression transformants. Mycelial plugs (5 $\mathrm{mm}$ in diameter) cut from the edge of 2-day-old colonies were transferred onto PDA dishes amended with dimethachlone as described above, and PDA dishes free of dimethachlone were used as the controls. After incubation at $23^{\circ} \mathrm{C}$ for 2 days, the diameter of each mycelial colony was measured twice at right angles. $\mathrm{EC}_{50}$ values were calculated according to Zhou et al. (2014a). The experiment was performed in triplicate and repeated independently four times.

Determination of sclerotial production and mycelial growth on PDA. Mycelial plugs ( $5 \mathrm{~mm}$ in diameter) cut from the edge of 2-day-old colonies were transferred to PDA dishes. Six replicate dishes of each isolate were incubated at $23^{\circ} \mathrm{C}$ for 30 days. During the first $60 \mathrm{~h}$ of incubation, colony diameters were measured once every $12 \mathrm{~h}$. The sclerotial production on PDA was recorded after 30 days of incubation. The experiment was repeated independently twice.

Virulence assays on detached leaves. The virulence assay was performed according to Li et al. (2015). For determination of virulence to cucumber (cultivar Xinjinyou 1) and oilseed rape (Zhongshuang 10), leaves of similar size and position were detached from approximately 35-day-old plants and transferred to $15-\mathrm{cm}$ Petri dishes lined with wet filter paper. The adaxial surfaces of the leaves were inoculated with mycelial plugs $(5 \mathrm{~mm}$ in diameter) cut from the fresh edge of 2-day-old colonies of each isolate and transformant. The diameter of each disease lesion was measured twice at right angles after 2 days of incubation $\left(23^{\circ} \mathrm{C}\right.$, $85 \%$ relative humidity, photoperiod of $16 \mathrm{~h}$ of light and $8 \mathrm{~h}$ of darkness) for the detached oilseed rape leaves and after 3 days of incubation for the detached cucumber leaves. Six replicates were used for each isolate in each of two independent experiments.

TABLE 1 . Polymerase chain reaction primers used in this study

\begin{tabular}{|c|c|c|}
\hline Primer & Sequence $\left(5^{\prime}-3^{\prime}\right)^{\mathrm{a}}$ & Relevant characteristics ${ }^{\mathrm{b}}$ \\
\hline SSHKF & CCCaagcttctgcagATGCGTGGAGATGTTTGGG & Amplify the silenced fragment of the $S s h k$ gene (306 bp) \\
\hline SSHKR & CCatcgatggatccCGAGTCCACAACGATCACATCATAA & \\
\hline PCIT-F & ACTAGAAGGCACTCTTTGCTGC & Confirm whether the $S s h k$ silenced fragment replaced the PCIT vector ( $630 \mathrm{bp}$ ) \\
\hline PCIT-F1 & TTCGTGGTCAAGGTCCATC & $\ldots$ \\
\hline PCIT-R1 & AGCAGACAGGAACGAGGAC & $\ldots$ \\
\hline OEHKF & CgagctcATGGGGGACACTACGATAGCTC & Amplify the overexpression fragment of the $S s h k$ gene $(3,920 \mathrm{bp})$ \\
\hline OEHKR & CCcccgggTTAGTACAGGTTCCTTGCAAGTGG & \\
\hline Rt-F & AAGGGTAGGGAAGTAAGGCAATC & Amplify the $S s h k$ gene for quantitative real-time PCR (153 bp) \\
\hline Rt-R & GTTTATAGGGCCAGTGGTAGTGTATG & $\ldots$ \\
\hline
\end{tabular}

${ }^{a}$ Lowercase letters represent the restriction enzyme sites in the primer sequence.

b $\mathrm{PCR}=$ polymerase chain reaction. 
Sensitivity to osmotic and oxidative stresses. For determination of sensitivity to sodium chloride $(\mathrm{NaCl})$, glucose, sucrose, glycerol, and hydrogen peroxide $\left(\mathrm{H}_{2} \mathrm{O}_{2}\right)$, mycelial plugs ( $5 \mathrm{~mm}$ in diameter) cut from the fresh edge of 2-day-old colonies of the parent isolate HLJ4 and the $S s h k$-silenced transformants were transferred onto PDA amended with $\mathrm{NaCl}$ at 5, 10, 20, 30, 40, 50, 60, 70 , and $80 \mathrm{~g} /$ liter; glucose at 10,20,40,60,80,100, 150, 200, and $300 \mathrm{~g} /$ liter; sucrose at 10, 50, 100, 150, 200, 250, 300, and $350 \mathrm{~g} /$ liter; glycerol at 5, 10, 20, 40, 80, 100, and $200 \mathrm{~g} /$ liter; or $\mathrm{H}_{2} \mathrm{O}_{2}$ at 5, $10,20,40,80,100,200,300$, and $400 \mathrm{mM}$. Mycelial plugs $(5 \mathrm{~mm}$ in diameter) cut from the fresh edge of 2-day-old colonies of the parent isolate HLJMG1 and the Sshk-overexpression transformants were transferred onto PDA amended with $\mathrm{NaCl}$ at 1.25, 2.5, 5, 10, 20, 30, 40, 50, 60, 70, 80, and $120 \mathrm{~g} /$ liter; glucose at 10, 20, 40, 60, 80, 100, 160, 320, and $500 \mathrm{~g} / \mathrm{liter}$; sucrose at 5, 10, 20, 40, 80, 100, 150, 200, and $400 \mathrm{~g} /$ liter; glycerol at 5, 10, 20, 40, 80, 100, 200, and $400 \mathrm{~g} / \mathrm{liter}$; or $\mathrm{H}_{2} \mathrm{O}_{2}$ at $5,10,20,40,80,100,200$, and $400 \mathrm{mM}$. PDA dishes without amendments were used as the controls. After incubation at $23^{\circ} \mathrm{C}$ for 2 days, the diameter of each mycelial colony was measured twice at right angles, and percent inhibition of mycelial growth relative to the controls was calculated. The experiment was performed in triplicate and repeated independently twice.

Cell wall and cell membrane integrity assays. Chitin is a crucial component of the cell wall of pathogenic fungi. Congo red (CR) interferes with the integrity of the cell wall by binding to chitin (Ram and Klis 2006). Sodium dodecyl sulfate (SDS) is an ionic surfactant that can dissolve membrane proteins and damage the cell membrane structure (Bulawa 1993). In this study, mycelial plugs ( $5 \mathrm{~mm}$ in diameter) cut from the fresh edge of 2-day-old colonies were transferred onto PDA amended with CR at $0.5,1,2,4,6$, and $8 \mathrm{mg} / \mathrm{ml}$ or SDS at $0.0025,0.005,0.0075,0.01,0.015,0.02,0.03$, and $0.04 \%$ (wt/vol). PDA media without CR or SDS was used as the
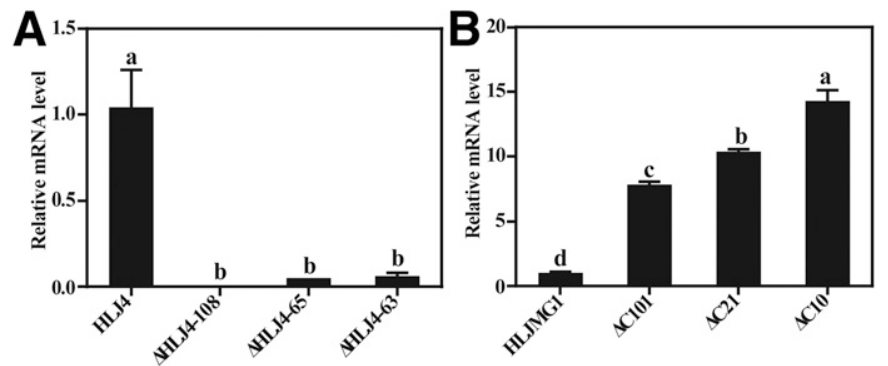

Fig. 1. Relative mRNA levels of $S s h k$ in A, the parent isolate HLJ4 of Sclerotinia sclerotiorum and its Sshk-silenced transformants and B, HLJMG1 and its $S$ shk-overexpression transformants. Bars denote the standard error of the mean of nine observations (three replicates in each of three independent experiments). Different lowercase letters above the bars indicate statistically significant differences $(\alpha=0.05)$.
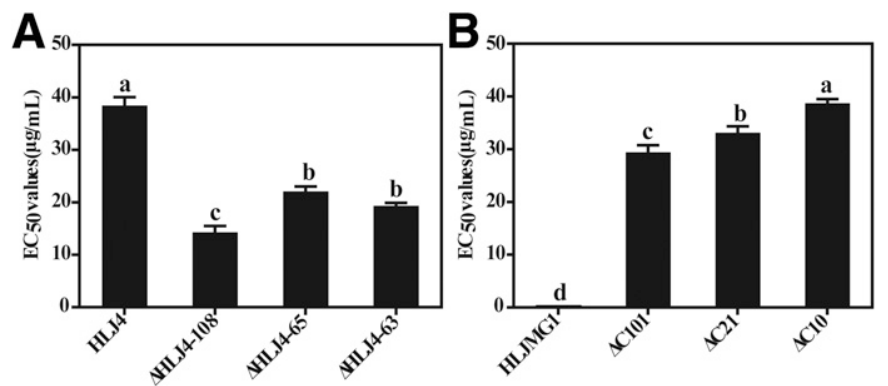

Fig. 2. Dimethachlone sensitivity in A, the parent isolate HLJ4 of Sclerotinia sclerotiorum and its Sshk-silenced transformants and B, HLJMG1 and its Sshkoverexpression transformants. $\mathrm{EC}_{50}$ values = effective concentrations for $50 \%$ inhibition of mycelial growth. Bars denote the standard error of the mean of 12 observations (three replicates in each of four independent experiments). Different lowercase letters above the bars indicate statistically significant differences $(\alpha=0.05)$. control. After incubation for $48 \mathrm{~h}$ at $23^{\circ} \mathrm{C}$, the diameter of each mycelial colony was measured twice at right angles, and percent inhibition of mycelial growth relative to the control was calculated. The experiment was performed in triplicate and repeated independently twice.

Transmission electron microscopy observations on hyphal cell wall morphology. Transmission electron microscopy (TEM) observations were according to Yu et al. (2012). Mycelia of the parent isolate HLJ4 and the $S s h k$-silenced transformants grown at $23^{\circ} \mathrm{C}$ for $48 \mathrm{~h}$ on cellophane-overlaid PDA were harvested by gentle scraping with a scoop. The samples were fixed at $4^{\circ} \mathrm{C}$ for $6 \mathrm{~h}$ in $4 \%(\mathrm{vol} / \mathrm{vol})$ glutaraldehyde in $100 \mathrm{mM}$ phosphate buffer $(\mathrm{pH}=7.2)$, thoroughly rinsed with phosphate buffer, postfixed with osmium tetroxide at $4^{\circ} \mathrm{C}$ for $2 \mathrm{~h}$, and dehydrated in a graded acetone series from 30 to $100 \%$. The samples were then embedded in Epon-821 resin (Hexion, Columbus, $\mathrm{OH}$ ) and polymerized at $60^{\circ} \mathrm{C}$ for $24 \mathrm{~h}$. Ultrathin sections $(50 \mathrm{~nm})$ were cut from the samples on a Leica Ultracut UCT ultramicrotome with a diamond knife and collected onto 200-mesh copper grids. After the contrasting procedure with uranyl acetate and lead citrate, the grids were examined with an electron microscope H-7650 (Hitachi, Tokyo) at $80 \mathrm{kV}$. The experiment was performed in triplicate and repeated independently twice.

Data analysis. A paired $t$ test was used to analyze data of lesion diameter and sclerotial production, and Fisher's least significant difference test following one-way analysis of variance in SPSS (ver. 17; SPSS Inc., Chicago) was used to evaluate significant differences in expression levels of the $S s h k$ gene and $\mathrm{EC}_{50}$ values of dimethachlone. A general linear model was used to evaluate significant differences in slopes of the fitted mycelial growth lines between the parent isolates and the transformants.

\section{RESULTS}

Transcriptional levels of $S s h k$ in the $S s h k$-silenced and -overexpression transformants. Compared with the parent isolate HLJ4, the transcription levels of $S s h k$ in the three silenced transformants $\Delta \mathrm{HLJ} 4-108, \Delta \mathrm{HLJ} 4-65$, and $\Delta \mathrm{HLJ} 4-63$ declined by $99.9,94.1$, and $95.1 \%$, respectively (Fig. 1A). Compared with the parent isolate HLJMG1, the $S s h k$ transcription levels in the three Sshk-overexpression transformants $\Delta \mathrm{C} 101, \Delta \mathrm{C} 21$, and $\Delta \mathrm{C} 10$ increased $(P<0.01) 7.7-, 10.2-$, and 14.1-fold, respectively (Fig. 1B).

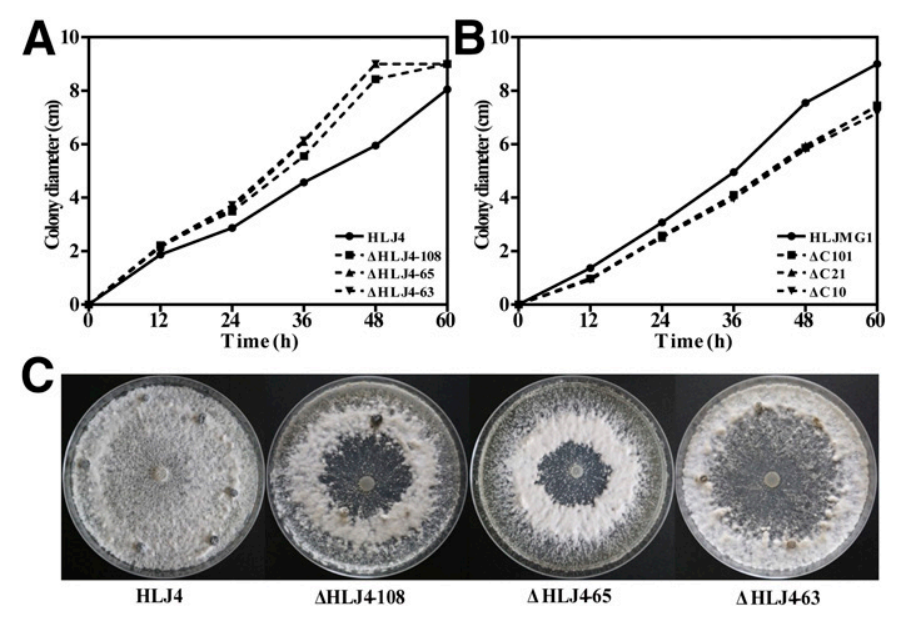

Fig. 3. Mycelial growth of A, the parent isolate HLJ4 of Sclerotinia sclerotiorum and its Sshk-silenced transformants and B, HLJMG1 and its Sshkoverexpression transformants; and C, sclerotial formation of the Sshk-silenced transformants on potato dextrose agar. Photos were taken after incubation for 30 days. Bars denote the standard error of the mean of 12 observations (six replicates in each of two independent experiments). 
Dimethachlone resistance in Sshk-silenced and -overexpression transformants. Compared with the parent isolate HLJ4, dimethachlone resistance ratios of the three $S s h k$-silenced transformants $\Delta \mathrm{HLJ} 4-108, \Delta \mathrm{HLJ} 4-65$, and $\Delta \mathrm{HLJ} 4-63$ declined by $62.9,42.5$, and $49.8 \%$, respectively, and $\mathrm{EC}_{50}$ values were 14.3, 22.1 , and $19.3 \mu \mathrm{g} / \mathrm{ml}$, respectively (Fig. 2A). Compared with the parent isolate HLJMG1, dimethachlone resistance ratios of the three overexpression transformants $\Delta \mathrm{C} 101, \Delta \mathrm{C} 21$, and $\Delta \mathrm{C} 10$ increased $(P<0.01)$ 168.1-, 189.5-, and 221.2-fold, respectively, and $\mathrm{EC}_{50}$ values were 29.4, 33.2, and $38.7 \mu \mathrm{g} / \mathrm{ml}$, respectively (Fig. 2B). These findings indicate that the transcriptional upregulation of the $S s h k$ gene is a major mechanism for dimethachlone resistance in some isolates of $S$. sclerotiorum.

Effects of Sshk expression on mycelial growth and sclerotial production. Compared with the parent isolate HLJ4, colony diameter of the $S s h k$-silenced transformants $\Delta$ HLJ4-108, $\triangle$ HLJ4-65, and $\triangle$ HLJ4-63 on PDA media increased (Fig. 3A), and there were significant differences in slopes of the fitted mycelial growth lines between the parent isolate and the $S s h k$-silenced transformants (the three $S s h k$-silenced transformants were used as three biological replicates). The silenced transformant $\Delta$ HLJ4-65 was incapable of producing sclerotia (Fig. 3C), and the number of sclerotia produced by the transformants $\Delta \mathrm{HLJ} 4-108$ and $\Delta \mathrm{HLJ} 4-63$ declined. Compared with the parent isolate HLJMG1, colony diameter of the three overexpression transformants $\Delta \mathrm{C} 101, \Delta \mathrm{C} 21$, and $\Delta \mathrm{C} 10$ decreased (Fig. 3B), and there were significant differences in slopes between the parent isolate and the overexpression transformants (the three overexpression transformants were used as three biological replicates), indicating that expression of the $S s h k$ gene affects mycelial growth and sclerotial production of S. sclerotiorum.

Effects of $\boldsymbol{S} \boldsymbol{s} \boldsymbol{k} \boldsymbol{k}$ expression on virulence. Compared with the parent isolate HLJ4, lesion diameters for the silenced transformants $\Delta$ HLJ4-108, $\Delta$ HLJ4-65, and $\triangle$ HLJ4-63 increased significantly $(P<$ $0.05)$ (Fig. 4). Lesion diameters for the overexpression transformants $\Delta \mathrm{C} 101, \Delta \mathrm{C} 21$, and $\Delta \mathrm{C} 10$ decreased significantly $(P<$ $0.05)$ compared with the parent isolate HLJMG1. These findings indicate that overexpression of $S s h k$ has a negative effect on the virulence of $S$. sclerotiorum.

Effects of $S$ shk expression on sensitivity to osmotic and oxidative stresses. Percent inhibitions of mycelial growth on PDA media supplemented with glucose (Fig. 5A), sucrose (Fig. 5B), $\mathrm{NaCl}$ (Fig. 5C), and glycerol (Fig. 5D) were lower for the $S$ shk-
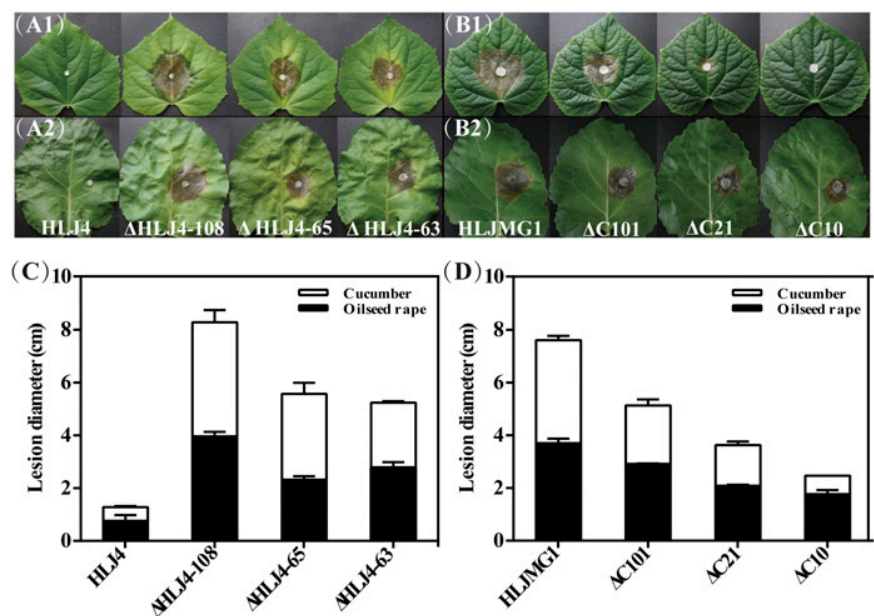

Fig. 4. Lesion development on detached leaves of A1 and B1, cucumber and $\mathbf{A 2}$ and B2, rapeseed 3 and 2 days, respectively, after inoculation with Sclerotinia sclerotiorum, and the diameters of disease lesions caused by the $\mathbf{C}$, $S s h k$-silenced transformants and $\mathbf{D}, S s h k$-overexpression transformants. Bars denote the standard error of the mean of 12 observations (six replicates in each of two independent experiments). silenced transformants compared with the parent isolate HLJ4, and higher for the overexpression transformants compared with the parent isolate HLJMG1. Percent inhibitions of mycelial growth on PDA amended with $\mathrm{H}_{2} \mathrm{O}_{2}$ at $200 \mathrm{mM}$ for the parent isolate $\mathrm{HLJ} 4$ and Sshk-silenced transformants were 100 and $71.8 \%$ (average), respectively. Percent inhibitions on PDA amended with $200 \mathrm{mM}$ $\mathrm{H}_{2} \mathrm{O}_{2}$ were 70.0 and $100 \%$ for the parent isolate HLJMG1 and $S$ shkoverexpression transformants, respectively (Fig. 5E). These results indicate that transcription levels of the $S s h k$ gene influence osmoadaptation and tolerance to oxidative stress in S. sclerotiorum.

Effects of $S s h k$ expression on the integrity of hyphal cell wall and cell membrane. Compared with the parent isolate HLJ4, percent inhibitions of mycelial growth on PDA amended with CR were significantly lower according to a paired $t$ test $(P<$ 0.05 ) for the three silenced transformants (Fig. 6A), indicating that the $S s h k$-silenced transformants were less sensitive to CR than the parent isolate. Compared with the parent isolate HLJMG1, percent inhibitions of mycelial growth on PDA supplemented with CR
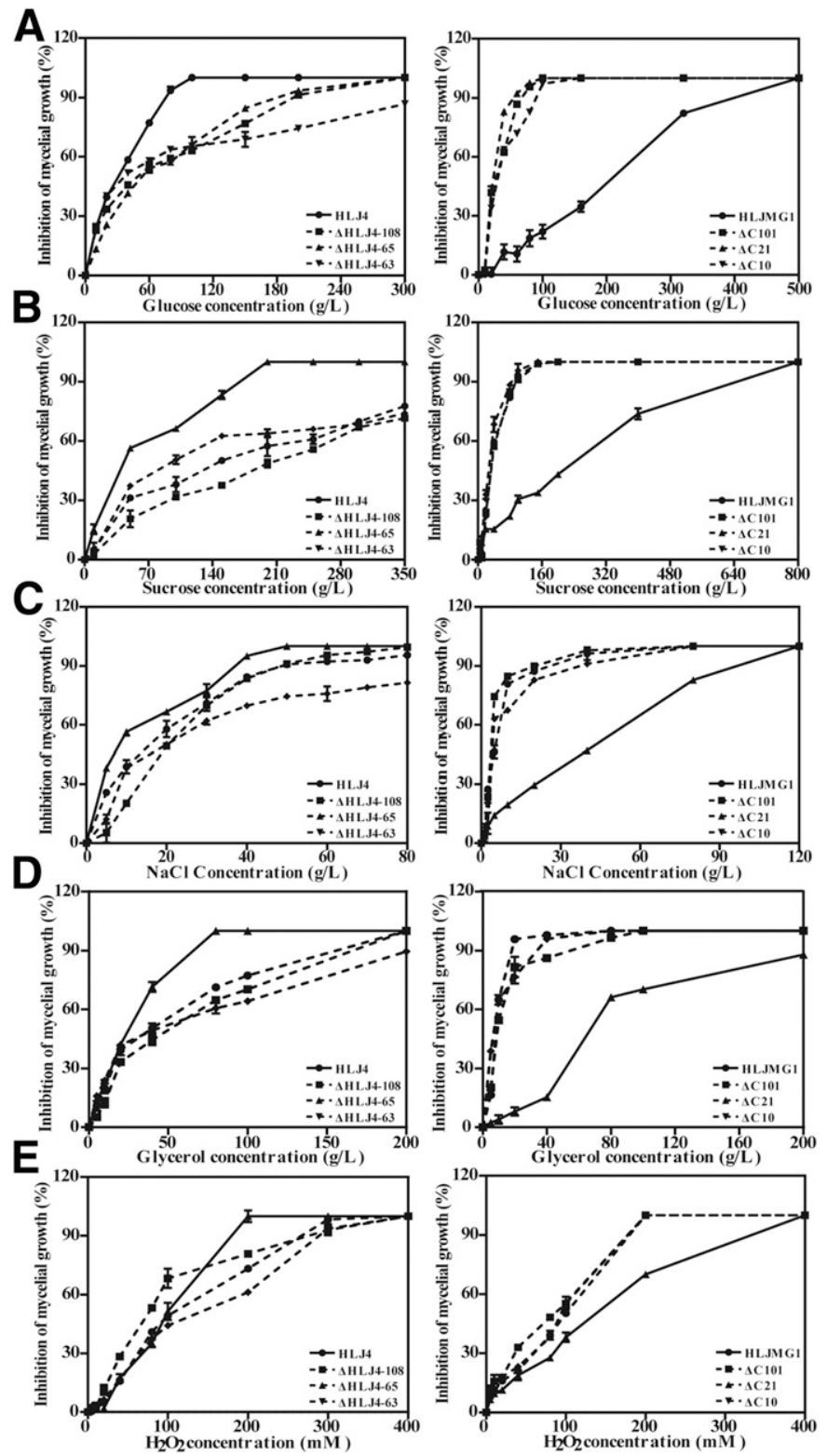

Fig. 5. Inhibition of mycelial growth of the parent isolates HLJ4 and HLJMG1 of Sclerotinia sclerotiorum and their Sshk-silenced or Sshk-overexpression transformants on potato dextrose agar amended with $\mathbf{A}$, glucose; B, sucrose; C, $\mathrm{NaCl} ; \mathbf{D}$, glycerol; and $\mathbf{E}, \mathrm{H}_{2} \mathrm{O}_{2}$. Bars denote the standard error of the mean of six observations (three replicates in each of two independent experiments). 
increased significantly according to a paired $t$ test $(P<0.05)$ for the three overexpression transformants (Fig. 6A). Percent inhibitions of mycelial growth on PDA supplemented with SDS for both the $S s h k$ silenced and -overexpression transformants increased significantly according to a paired $t$ test $(P<0.05)$ compared with their respective parent isolates (Fig. 6B). TEM observations indicated that the cell wall and plasma membrane of most cells shrank for the Sshksilenced transformants, the organelles of some cells were destroyed and the contents leaked in the transformants $\Delta$ HLJ4-108 and $\Delta$ HLJ4-65, and the organelles of almost every cell collapsed in $\Delta$ HLJ4-63, leading to cell lysis (Fig. 7). These results suggest that adequate expression of the gene $S s h k$ is necessary for maintaining the integrity of the cell wall and cell membrane.

\section{DISCUSSION}

Mutations or deletions of group III HK genes were previously shown to mediate resistance to DCF, phenylpyrrole, and aromatic hydrocarbon fungicides (Cui et al. 2002; Duan et al. 2013; Luo et al. 2012; Motoyama et al. 2005; Ochiai et al. 2001; Oshima et al. 2002; Yoshimi et al. 2004). Our previous studies demonstrated that the field dimethachlone-resistant isolates have no cross resistance with the phenylpyrrole fungicide fludioxonil (Zhou et al. 2014a), and expression levels of the group III HK gene Sshk are higher in the field isolates than in the sensitive isolates of $S$. sclerotiorum (Li et al. 2017). In this study, therefore, we constructed group III Sshksilencing and -overexpression vectors and demonstrated that silencing of $S s h k$ led to a significant decrease in dimethachlone resistance and overexpression of $S s h k$ resulted in a dramatic increase in dimethachlone resistance. The transcription levels of Sshk in three silenced transformants were downregulated by 99.9 , 94.1 , and $95.1 \%$, respectively, and dimethachlone resistance ratios declined by $62.9,42.5$, and $49.8 \%$, respectively. The silenced transformants still retained medium levels of dimethachlone resistance. Compared with the dimethachlone-sensitive parent isolate HLJMG1, the Sshk transcription levels of three overexpression transformants increased 7.7-, 10.2-, and 14.1-fold, respectively, and the dimethachlone resistance ratio increased 168.1-, 189.5-, and 221.2-fold, respectively. According to a recent study, lost expression of a group III HK gene $\mathrm{Ss} O s \mathrm{I}$ may be involved in dimethachlone resistance in S. sclerotiorum (Firoz et al. 2016). These results indicate that different mechanisms exist for target resistance to DCF, and transcriptional upregulation of $S s h k$ is a
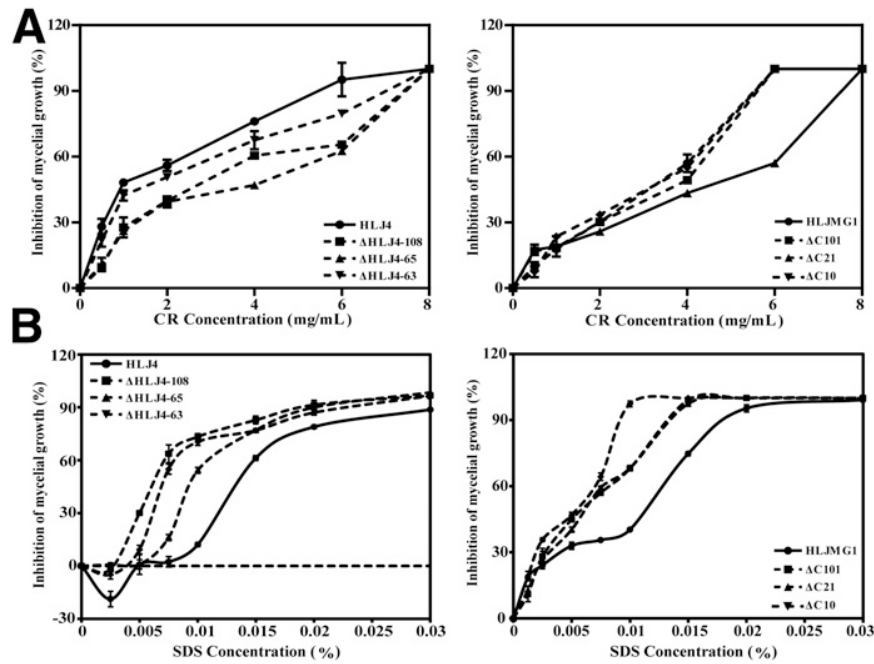

Fig. 6. Inhibition of mycelial growth of the parent isolates HLJ4 and HLJMG1 of Sclerotinia sclerotiorum and their Sshk-silenced or Sshk-overexpression transformants on potato dextrose agar amended with A, Congo red (CR) and B, sodium dodecyl sulfate (SDS). Bars denote the standard error of the mean of six observations (three replicates in each of two independent experiments). novel mechanism for dimethachlone resistance in some isolates of $S$. sclerotiorum. This is one case of overexpression of the target protein as a resistance mechanism and corroborates that the action targets of the DCF are the group III HK in the two-component signal pathway.

Biological characteristics, including mycelial growth, sclerotium formation, and osmotic response, have been reported in HKdeletion mutants of phytopathogenic fungi (Duan et al. 2013; Firoz et al. 2016; Luo et al. 2012; Rispail and Pietro 2010; Zhang et al. 2010). Group III HK play different roles in virulence of different fungal species with different genetic backgrounds. For example, the genes Shkl and HIKl are not required for virulence of S. sclerotiorum and P. oryzae (Duan et al. 2013; Motoyama et al. 2005) but are required in Fusarium oxysporum and Botrytis cinerea (Rispail and Pietro 2010; Viaud et al. 2006). In this study, we demonstrated that overexpression of $S s h k$ resulted in resistance to dimethachlone, increased sensitivity to hyperosmotic and oxidative stresses, and reduced virulence. For $S$. sclerotiorum, sclerotia play a major role in long-term survival (Willetts and Wong 1980), and mycelial growth is a primary indicator of aggressiveness (Garg et al. 2010). Our results showed that the $S$ shk-silenced transformants had increased mycelial growth but reduced sclerotium-production capability and shriveled sclerotia. These results indicate that transcription of $S s h k$ is involved in sclerotium development and vegetative differentiation in $S$. sclerotiorum.

The fungal cell wall regulates responses of fungal cells to environmental stimuli (Jeon et al. 2008), and chitin is an essential component of the fungal cell wall. The anionic dye CR can combine with chitin, resulting in changes in hyphal morphology such as hyphal tips lysis, cell separation, or activation of responses of the cell wall to stresses (Boorsma et al. 2004; Damveld et al. 2005a,b; García et al. 2004; Lagorce et al. 2003; Pancaldi et al. 1984; Roncero and Duran 1985; Vannini et al. 1983). In P. oryzae, the $\Delta M o s \ln 1$ mutant has increased resistance to the cell wall stressor Calcofluor white (Zhang et al. 2010). In the present study, the Sshksilenced transformants became less sensitive to CR, indicating an increase in chitin contents in the cell wall of $S$. sclerotiorum. Interestingly, we also found that the cell walls of most cells were

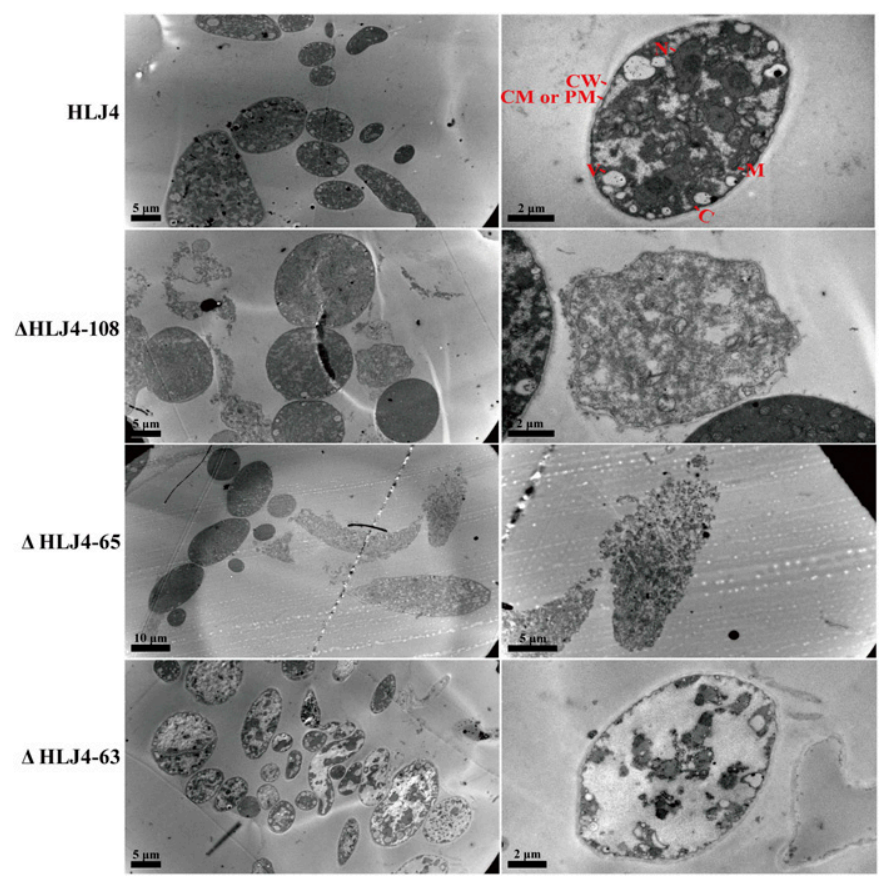

Fig. 7. Microscopic structures of the parent isolate HLJ4 of Sclerotinia sclerotiorum and $S$ shk-silenced transformants observed by transmission electron microscopy. $\mathrm{CW}=$ cell wall, $\mathrm{CM}=$ cell membrane, $\mathrm{PM}=$ plasma membrane, $\mathrm{V}=$ vacuole, $\mathrm{C}=$ cytoplasm, $\mathrm{M}=$ mitochondrion, and $\mathrm{N}=$ nucleus. 
damaged and the organelles of some cells collapsed in the Sshksilenced transformants.

In conclusion, the present study demonstrated that transcriptional upregulation of the group III HK gene $S s h k$ was a vital mechanism for dimethachlone resistance in some isolates of S. sclerotiorum. The transcription levels of $S s h k$ had a great impact on fitness-related parameters of $S$. sclerotiorum. Overexpression of $S$ shk reduced mycelial growth and virulence to host plants while it increased sensitivity of $S$. sclerotiorum to exogenous stresses. The fitness costs associated with dimethachlone resistance may account for the slow development of dimethachlone resistance in the field. These findings will advance our understanding of the mechanisms of resistance to the dicarboximide fungicides in S. sclerotiorum and should be taken into account in designing resistance management strategies.

\section{ACKNOWLEDGMENTS}

We thank J. Xie (College of Plant Science and Technology, Huazhong Agricultural University) for kindly providing vectors (pCIT, $\mathrm{pCH}$, and pCETNS-4)

\section{LITERATURE CITED}

Alex, L. A., Borkovich, K. A., and Simon, M. I. 1996. Hyphal development in Neurospora crassa: Involvement of a two-component histidine kinase. Proc. Natl. Acad. Sci. USA 93:3416-3421.

Bardin, S. D., and Huang, H. C. 2001. Research on biology and control of Sclerotinia diseases in Canada. Can. J. Plant Pathol. 23:88-98.

Bolton, D. M., Thomma, B. P., and Nelson, B. D. 2006. Sclerotinia sclerotiorum (lib.) de Bary: Biology and molecular traits of a cosmopolitan pathogen. Mol. Plant Pathol. 7:1-16.

Boorsma, A., de Nobel, H., ter Riet, B., Bargmann, B., Brul, S., Hellingwerf, K. J., and Klis, F. M. 2004. Characterization of the transcriptional response to cell wall stress in Saccharomyces cerevisiae. Yeast 21:413-427.

Bulawa, C. E. 1993. Genetics and molecular biology of chitin synthesis in fungi. Annu. Rev. Microbiol. 47:505-534.

Calera, J. A., Zhao, X. J., and Calderone, R. 2000. Defective hyphal development and avirulence caused by a deletion of the SSK1 response regulator gene in Candida albicans. Infect. Immun. 68:518-525.

Catlett, N. L., Yoder, O. C., and Turgeon, B. G. 2003. Whole-genome analysis of two component signal transduction genes in fungal pathogens. Eukaryot. Cell 2:1151-1161.

Cho, Y., Kim, K. H., La, R. M., Scott, D., Santopietro, G., Callihan, M., Mitchell, T. K., and Lawrence, C. B. 2009. Identification of novel virulence factors associated with signal transduction pathways in Alternaria brassicicola. Mol. Microbiol. 72:1316-1333.

Cui, W., Beever, R. E., Parkes, S. L., Weeds, P. L., and Templetona, M. D. 2002. An osmosensing histidine kinase mediates dicarboximide fungicide resistance in Botryotinia fuckeliana (Botrytis cinerea). Fungal Genet. Biol. 36:187-198.

Damveld, R. A., Arentshorst, M., Franken, A., vanKuyk, P. A., Klis, F. M., van den Hondel, C. A., and Ram, A. F. 2005a. The Aspergillus niger MADS-box transcription factor $R \operatorname{lmA}$ is required for cell wall reinforcement in response to cell wall stress. Mol. Microbiol. 58:305-319.

Damveld, R. A., vanKuyk, P. A., Arentshorst, M., Klis, F. M., van den Hondel, C. A., and Ram, A. F. 2005b. Expression of agsA, one of five 1,3-alpha-Dglucan synthase encoding genes in Aspergillus niger, is induced in response to cell wall stress. Fungal Genet. Biol. 42:165-177.

Duan, Y. B., Ge, C. Y., Liu, S. M., Wang, J. X., and Zhou, M. G. 2013. A two component histidine kinase Shkl controls stress response, sclerotial formation and fungicide resistance in Sclerotinia sclerotiorum. Mol. Plant Pathol. 14:708-718.

Firoz, M. J., Xiao, X., Zhu, F. X., Fu, Y. P., Jiang, D. H., Schnabel, G., and Luo, C. X. 2016. Exploring mechanisms of resistance to dimethachlone in Sclerotinia sclerotiorum. Pest Manage. Sci. 72:770-779.

García, R., Bermejo, C., Grau, C., Pérez, R., Rodríguez-Peña, J. M., Francois, J., Nombela, C., and Arroyo, J. 2004. The global transcriptional response to transient cell wall damage in Saccharomyces cerevisiae and its regulation by the cell integrity signaling pathway. J. Biol. Chem. 279: 15183-15195.

Garg, H., Kohn, L. M., Andrew, M., Li, H., Sivasithamparam, K., and Barbetti, M. J. 2010. Pathogenicity of morphologically different isolates of Sclerotinia sclerotiorum with Brassica napus and B. juncea genotypes. Eur. J. Plant Pathol. 126:305-315.

Izumitsu, K., Kobayashi, H., Morita, A., Saitoh, Y., and Tanaka, C. 2010. Twocomponent signaling system is important for osmotic adaptation, fungicidal sensitivity, morphologenesis, and pathogenicity in Botrytis cinerea. In: Proc. 9th Int. Mycol. Congr., Edinburgh.

Jeon, J., Goh, J., Yoo, S., Chi, M. H., Choi, J., Rho, H. S., Park, J., Han, S. S., Kim, B. R., Park, S. Y., Kim, S., and Lee, Y. H. 2008. A putative MAP kinase kinase kinase, $M C K 1$, is required for cell wall integrity and pathogenicity of the rice blast fungus, Magnaporthe oryzae. Mol. Plant-Microbe Interact. 21:525-534.

Jiang, M. 2007. Establishment of mycelium transformation system of Sclerotinia sclerotiorum with Agrobacterium tumefaciens and preliminary study on the function of Ss-CYP1 downregulated by mycovirus SsDRV. Master's dissertation, Huazhong Agricultural University, Wu Han, China.

Kuang, J., Wang, J., and Zhou, M. 2011. Monitoring on carbendazim and dimethachlone resistance of Sclerotinia sclerotiorum obtained from the blight stems of rape in Jiangsu Province. Chin. Agric. Sci. Bull. 27:285-291.

Lagorce, A., Hauser, N. C., Labourdette, D., Rodriguez, C., Yken, H. M., Arroyo, J., Hoheisel, J. D., and Francois, J. 2003. Genome-wide analysis of the response to cell wall mutations in the yeast Saccharomyces cerevisiae. J. Biol. Chem. 278:20345-20357.

Letham, D. B., Huett, D. O., and Trimboli, D. S. 1976. Biology and control of Sclerotinia sclerotiorum in cauliflower and tomato crops in coastal New South Wales. Plant Dis. 60:286-289.

Li, J. L., Kang, T. H., Talab, K. M. A., Zhu, F. X., and Li, J. H. 2017. Molecular and biochemical characterization of dimethachlone resistant isolates of Sclerotinia sclerotiorum. Pestic. Biochem. Physiol. 138:15-21.

Li, J. L., Wu, F. C., and Zhu, F. X. 2015. Fitness is recovered with the decline of dimethachlon resistance in laboratory induced mutants of Sclerotinia sclerotiorum after long-term cold storage. Plant Pathol. J. 31:305-309.

Li, S., Ault, A., Malone, C. L., Raitt, D., Dean, S., Johnston, L. H., Deschenes, R. J., and Fassler, J. S. 1998. The yeast histidine protein kinase, Sln1p, mediates phosphotransfer to two response regulators, $S s k 1 p$ and $S k n 7 p$. EMBO J. 17:6952-6962.

Liu, W., Leroux, P., and Fillinger, S. 2008. The HOG1-like MAP kinase Sak1 of Botrytis cinerea is negatively regulated by the upstream histidine kinase Bos 1 and is not involved in dicarboximide- and phenylpyrrole-resistance. Fungal Genet. Biol. 45:1062-1074.

Livak, K. J., and Schmittgen, T. D. 2001. Analysis of relative gene expression data using real-time quantitative PCR and the $2^{-\Delta \Delta C T}$ method. Methods 25: 402-408.

Luo, Y. Y., Yang, J. K., Zhu, M. L., Liu, C. J., Li, H. Y., Lu, Z. B., Pan, W. Z., Zhang, Z. H., Bi, W., and Zhang, K. Q. 2012. The group III two-component histidine kinase $A l H K 1$ is involved in fungicides resistance, osmosensitivity, spore production and impacts negatively pathogenicity in Alternaria longipes. Curr. Microbiol. 64:449-456.

Ma, H. X., Feng, X. J., Chen, Y., Chen, C. J., and Zhou, M. G. 2009. Occurrence and characterization of dimethachlone insensitivity in Sclerotinia sclerotiorum in Jiangsu province of China. Plant Dis. 9:336-342.

Motoyama, T., Kodama, K., Ohira, T., Ichiishi, A., Fujimura, M., Yamaguchi, I., and Kubo, T. 2005. A two-component histidine kinase of the rice blast fungus is involved in osmotic stress response and fungicide action. Fungal Genet. Biol. 42:200-212.

Ochiai, N., Fujimura, M., Motoyama, T., Ichiishi, A., Usami, R., Horikoshi, K., and Yamaguchi, I. 2001. Characterization of mutations in the two-component histidine kinase gene that confer fludioxonil resistance and osmotic sensitivity in the os-1 mutants of Neurospora crassa. Pest Manage. Sci. 57:437-442.

Ochiai, N., Fujimura, M., Oshima, M., Motoyama, T., Ichiishi, A., Yamada-Okame, H., and Yamaguchi, I. 2002. Effects of iprodione and fludioxonil on glycerol synthesis and hyphal development in Candida albicans. Biosci. Biotechnol. Biochem. 66:2209-2215.

Oshima, M., Fujimura, M., Banno, S., Hashimoto, C., Motoyama, T., Ichiishi, A., and Yamaguchi, I. 2002. A point mutation in the two-component histidine kinase $B c O S-1$ gene confers dicarboximide resistance in field isolates of Botrytis cinerea. Phytopathology 92:75-80.

Pan, Y. L. 1998. The resistance of Sclerotinia sclerotiorum of rape to carbendazim and its management. Jiangsu Agric Sci. 14:159-163.

Pancaldi, S., Poli, F., Dall'Olio, G., and Vannini, G. L. 1984. Morphological anomalies induced by Congo red in Aspergillus niger. Arch. Microbiol. 137:185-187.

Posas, F., Wurgler-Murphy, S. M., Maeda, T., Witten, E. A., Thai, T. C., and Saito, H. 1996. Yeast HOG1 MAP kinase cascade is regulated by a multistep phosphorelay mechanism in the SLN1-YPD1-SSK1 "two-component" osmosensor. Cell 86:865-875.

Ram, A. F. J., and Klis, F. M. 2006. Identification of fungal cell wall mutants using susceptibility assays based on Calcofluor white and Congo red. Nat. Protoc. 1:2253-2256.

Rispail, N., and Pietro, D. A. 2010. The two-component histidine kinase Fhk1 controls stress adaptation and virulence of Fusarium oxysporum. Mol. Plant Pathol. 11:395-407.

Roncero, C., and Duran, A. 1985. Effect of Calcofluor white and Congo red on fungal cell wall morphogenesis: In vivo activation of chitin polymerization. J. Bacteriol. 163:1180-1185. 
Srikantha, T., Tsai, L., Daniels, K., Enger, L., Highley, K., and Soll, D. R. 1998. The two-component hybrid kinase regulator CaNIK1 of Candida albicans. Microbiology 144:2715-2729.

Tanaka, C., and Izumitsu, K. 2010. Two-component signaling system in filamentous fungi and the mode of action of dicarboximide and phenylpyrrole fungicides. Pages 523-538 in: Fungicides. O. Crisse, ed. InTech, Rijeka, Croatia.

Thomason, P., Traynor, D., and Kay, R. 1999. Taking the plunge: Terminal differentiation in Dictyostelium. Trends Genet. 15:15-19.

Vannini, G. L., Poli, P., Donini, A., and Pancaldi, S. 1983. Effects of Congo red on wall synthesis and morphogenesis in Saccharomyces cerevisiae. Plant Sci. Lett. 31:9-17.

Viaud, M., Fillinger, S., Liu, W., Polepalli, J. S., Pecheur, L. P., Kunduru, A. R., Leroux, P., and Legendre, L. 2006. A class III histidine kinase acts as a novel virulence factor in Botrytis cinerea. Mol. Plant-Microbe Interact. 19:1042-1050.

West, A. H., and Stock, A. M. 2001. Histidine kinases and response regulator proteins in two component signaling systems. Trends Biochem. Sci. 26:369-376.

Willetts, H. J., and Wong, J. A. 1980. The biology of Sclerotinia sclerotiorum, $S$. trifoliorum, and $S$. minor with emphasis on specific nomenclature. Bot. Rev. 46:101-165.

Wurgler-Murphy, S. M., and Saito, H. 1997. Two-component signal transducers and MAPK cascades. Trends Biochem. Sci. 22:172-176.
Yang, G. G., Tang, L. G., Gong, Y. D., Xie, J. T., Fu, Y. P., Jiang, D. H., Li, G. Q., Collinge, D. B., Chen, W. D., and Cheng, J. S. 2018. A ceratoplatanin protein $\mathrm{SsCP} 1$ targets plant PR1 and contributes to virulence of Sclerotinia sclerotiorum. New Phytol. 217:739-755.

Yoshimi, A., Tsuda, M., and Tanaka, C. 2004. Cloning and characterization of the histidine kinase gene Dicl from Cochliobolus heterostrophus that confers dicarboximide resistance and osmotic adaptation. Mol. Genet. Genomics 271:228-236.

Yu, Y., Jiang, D. H., Xie, J. T., Cheng, J. S., Li, G. Q., Yi, X. H., and Fu, Y. P. 2012. Ss-S12, a novel cell wall protein with PAN modules, is essential for sclerotial development and cellular integrity of Sclerotinia sclerotiorum. PLoS One 7:e34962.

Zhang, H. F., Liu, K. Y., Zhang, X., Song, W. W., Zhao, Q., Dong, Y. H., Guo, M., Zheng, X. B., and Zhang, Z. G. 2010. A two-component histidine kinase, MoSLN1, is required for cell wall integrity and pathogenicity of the rice blast fungus Magnaporthe oryzae. Curr. Genet. 56:517-528.

Zhou, F., Zhang, X. L., Li, J. L., and Zhu, F. X. 2014a. Dimethachlone resistance in Sclerotinia sclerotiorum in China. Plant Dis. 98:1221-1226.

Zhou, F., Zhu, F. X., Zhang, X. L., and Zhang, A. S. 2014b. First report of dimethachlone resistance in field isolates of Sclerotinia sclerotiorum on oilseed rape in Shaanxi province of northwestern China. Plant Dis. 98: 568 